\title{
Questionários e escalas úteis na pesquisa da síndrome da apneia obstrutiva do sono
}

\author{
Maria Helena de Araújo-Melo, ${ }^{1,2,3 *}$ Denise D. Nevess, ${ }^{2,3,4}$ Lucas V. M. V. Ferreira, ${ }^{4}$ Marlos L. V. Moreira, ${ }^{5}$ Rafael \\ Nigri, ${ }^{5}$ Sílvia M. G. Simões ${ }^{5}$
}

\section{Resumo}

A síndrome da apneia obstrutiva do sono (SAOS) é uma condição clínica muito prevalente na população, com diversas repercussões clínicas e de saúde pública. Porém, o exame de Polissonografia (PSG), o padrão-ouro na confirmação da SAOS, não é utilizado de forma eficaz na sua rotina diagnóstica, principalmente na assistência em hospitais públicos brasileiros devido a sua pouca disponibilidade. Por serem de simples aplicação, diversas escalas e questionários relacionados ao sono podem ser utilizados para um diagnóstico presuntivo com efetiva triagem quando há suspeita da SAOS. Apesar do conhecimento de inúmeras outras escalas relacionadas a problemas de sono, vamos aqui descrever aquelas mais utilizadas no Laboratório de Sono da Universidade Federal do Estado do Rio de Janeiro (UNIRIO), como a Escala de Sonolência de Stanford (ESS), a Escala de Sonolência Diurna de Epworth (ESDE), a Escala do Ronco de Stanford (ERS), o Questionário de Berlim (QB), o questionário STOP-BANG e o questionário Sleep Apnea Quality of Life Index (SAQLI). Estes todos são instrumentos valiosos não só para uma triagem da SAOS como para a realização de pesquisas quantitativas e qualitativas na área da medicina do sono.

Descritores: Sensibilidade e especificidade; Diagnóstico; Apneia do sono tipo obstrutiva; Polissonografia; Transtornos do sono.

\section{Abstract \\ Questionnaires and scales useful in obstructive sleep apnea syndrome research}

Obstructive apnea syndrome (OSAS) is a very common medical condition in the population with distinct clinical and public health implications. Polysomnography (PSG), the gold standard test for OSAS confirmation, is not usual in routine practice, particularly at Brazilian public hospitals due to its limited availability. Because they are simple to apply, various scales and questionnaires related to sleep can be used for presumptive diagnosis with effective screening when there is suspicion of OSAS. We here describe the most commonly questionnaires used at the Sleep Laboratory of the State Federal University of Rio de Janeiro (UNIRIO) as the Stanford Sleepiness Scale (ESS), the Scale Daytime sleepiness Epworth (ESDE), the Stanford Snoring Scale (ERS), the Berlin Questionnaire (BQ), the STOP-BANG questionnaire and the questionnaire Sleep Apnea Quality of Life Index (SAQLI). These are all valuable tools not only for a screening of OSAS as for conducting quantitative and
1. Serviço de Otorrinolaringologia. Hospital Universitário Gaffrée e Guinle. Universidade Federal do Estado do Rio de Janeiro. Rio de Janeiro, RJ, Brasil.

2. Escola de Medicina e Cirurgia. Universidade Federal do Estado do Rio de Janeiro. Rio de Janeiro, RJ, Brasil.

3. LabSono. Hospital Universitário Gaffrée e Guinle. Universidade Federal do Estado do Rio de Janeiro. Rio de Janeiro, RJ, Brasil.

4. Serviço de Pneumologia. Hospital Universitário Gaffrée e Guinle. Universidade Federal do Estado do Rio de Janeiro. Rio de Janeiro, RJ, Brasil.

5. Bolsa de Iniciação Científica. LabSono. Hospital Universitário Gaffrée e Guinle. Universidade Federal do Estado do Rio de Janeiro. Rio de Janeiro, RJ, Brasil.

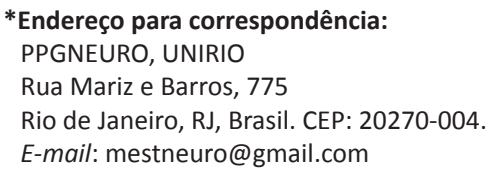

Revista HUPE, Rio de Janeiro, 2016;15(1):49-55

doi: 10.12957/rhupe.2016.22368

Recebido em 11/01/2015. Aprovado em 18/03/2016.

qualitative research in the field of sleep medicine.

Keywords: Sensitivity and specificity; Diagnosis; Obstructive sleep apnea; Polysomnography; Sleep disorders.

\section{Resumen}

Los cuestionarios y escalas de utilidad en la investigación del síndrome de apnea obstructiva del sueño

Síndrome del apnea obstructiva del sueño (SAOS) es una enfermedad muy común en la población con distintas implicaciones clínicas y de salud pública. Pero la prueba de polisomnografía (PSG), el estándar de oro para la confirmación de SAOS no se utiliza de manera efectiva en su práctica habitual, sobre todo en la asistencia en los hospitales públicos de Brasil, debido a su limitada disponibilidad. Debido a que son fáciles de aplicar, diversas escalas y cuestionarios relacionados con el sueño pueden ser utilizados para un diagnóstico presuntivo con el cribado eficaz cuando hay sospecha de SAOS. A pesar del conocimiento de numerosas otras escalas relacionadas con los problemas del sueño, que aquí describimos los más utilizados en el Laboratorio del Sueño de la Universidad Federal del Estado de Río de Janeiro (UNIRIO) como la escala 
de somnolencia de Stanford (ESS), la escala la somnolencia diurna de Epworth (ESDE), el Stanford ronquido Escala (ERS), el Cuestionario de Berlín (BQ), el cuestionario STOP-BANG y el cuestionario de la apnea del sueño Índice de Calidad de Vida (SAQLI). Todos estos son herramientas valiosas no sólo para la proyección de SAOS como para la realización de la

\section{Introdução}

Na avaliação clínica dos pacientes com queixas sugestivas de Síndrome da Apneia Obstrutiva do Sono (SAOS), diversas escalas e questionários são utilizados para fins de diagnóstico e de triagem, devido ao alto custo, complexidade e baixa ou pouca disponibilidade da Polissonografia (PSG), padrão-ouro para diagnóstico. ${ }^{1-3} \mathrm{Em} 3$ ou 4 minutos (o tempo para rever dez escalas padronizadas), um clínico pode chegar a uma compreensão ampla do paciente em questão. ${ }^{4}$

Há inúmeras escalas relacionadas aos problemas de sono. ${ }^{4} \mathrm{O}$ objetivo desta revisão é descrever aquelas consideradas mais utilizadas na prática clínica do Laboratório de Sono (LabSono) da Universidade Federal do Estado do Rio de Janeiro (UNIRIO), localizado no Hospital Universitário Gaffrée e Guinle (HUGG), Rio de Janeiro.

\section{Escala de Sonolência de Stanford (ESS)}

Uma das mais antigas escalas é uma medida subjetiva de sonolência, frequentemente usada tanto para fins de pesquisa como na prática clínica. Considerando que um instrumento como a Escala de Sonolência de Epworth, que veremos a seguir, examina experiências gerais de sonolência ao longo de um dia inteiro, a ESS avalia sonolência em momentos específicos no tempo. É constituida de um único item no qual o entrevistado seleciona uma das sete melhores situações que representa sua percepção do nível de sonolência. Como uma medida de item único, a escala é mais adequada para utilização repetida ao longo do estudo de investigação ou tratamento intervenção. Pode ser utilizada em qualquer população, mas é melhor avaliada em adultos acima de 18 anos. ${ }^{4}$ É uma escala autoadministrada em cerca de 1 a 2 minutos. ${ }^{4}$

Nessa escala, o paciente se enquadra entre as opções de 1 a 7, de acordo com o nível de sonolência, conforme ilustrado na tabela 1 .

Indivíduos que marcam 4, 5, 6 ou 7, em horas do dia em que deveriam estar acordados e alertas, podem ter sonolência diurna. Uma vantagem desta escala, visto que pode ser administrada várias vezes no mesmo dia investigación cuantitativa y cualitativa en el campo de la medicina del sueño.

Palabras clave: Sensibilidad y especificidad; Diagnóstico; Apnea del sueño obstructiva; Polissomnografía; Trastornos del sueño.

e em dias subsequentes, é poder diferenciar o nível de sonolência, diminuindo erros de aferição por situações atípicas. Além disso, ela possui uma boa correlação com privação do sono aguda. Sua maior desvantagem está na incapacidade de diferenciação entre indivíduos que têm privação do sono e indivíduos que estão fadigados ou cansados. ${ }^{5}$

\section{Escala de Sonolência de Epworth (ESE)}

Esta escala foi projetada para avaliar o grau de sonolência diurna de uma pessoa. ${ }^{6}$ De natureza simples, podendo ser autoaplicável, é composta por 8 situações do cotidiano nas quais o paciente é proposto a dar uma nota de 0 a 3 para a sua chance de cochilar ao executar tais situações, sendo que: 0 denota nenhuma chance de cochilar; a nota 1 se refere a uma chance pequena; a nota 2 corresponde a uma chance moderada; e a nota 3 consiste em uma alta probabilidade de cochilar.? Pode variar, portanto, de 0 a 24 pontos, sendo que uma pontuação acima de 10 sugere a ocorrência de sonolência diurna excessiva. ${ }^{8}$ Ao pesquisarmos "Epworth Sleepiness Scale" na base Pubmed, em 2014, encontramos 1.815 artigos publicados nos últimos 10

\section{Tabela 1. Escala de Sonolência de Stanford.}

\begin{tabular}{|c|c|}
\hline Grau de Sonolência & Nível \\
\hline Sente-se ativo, alerta, bem-disposto & 1 \\
\hline $\begin{array}{l}\text { Pode se concentrar, mas não está no nível } \\
\text { máximo de alerta }\end{array}$ & 2 \\
\hline $\begin{array}{l}\text { Relaxado, acordado e responsivo, mas não está } \\
\text { completamente alerta }\end{array}$ & 3 \\
\hline Sonolento, mas acordado & 4 \\
\hline $\begin{array}{l}\text { Sonolento quase dormindo, sem interesse em } \\
\text { permanecer acordado, pensamento lento }\end{array}$ & 5 \\
\hline Prefere estar deitado, luta contra o sono & 6 \\
\hline $\begin{array}{l}\text { Encontra-se perdendo a luta contra o sono, na } \\
\text { iminência do sono }\end{array}$ & 7 \\
\hline
\end{tabular}




\section{Artigo de revisão}

anos, enquanto "Epworth", na plataforma Scielo, na mesma data, o encontrado foi de 111 artigos nos últimos 10 anos, o que corrobora a ampla utilização desta escala por diversos estudos para avaliação de sonolência, provavelmente por sua natureza simples e fácil de ser aplicada. O questionário completo traduzido para o português encontra-se descrito na tabela 2 .

A partir de sua descrição, a Escala de Epworth passou a ser traduzida para vários idiomas e, em 2009, sua tradução para o português foi validada por um estudo realizado na Universidade Federal do Rio Grande do Sul (UFRGS), demonstrando que nenhum dos oito itens originais teve de ser modificado em relação ao seu conteúdo original e que a tradução para o português apresentou resultados semelhantes à Escala original, comparadas com resultados de PSG na população do estudo. ${ }^{9}$ A partir desse momento, tornou-se facilitada a realização de estudos com o uso desta escala no Brasil, fato exemplificado quando se nota que, dos 111 artigos da base Scielo citados anteriormente, 74 foram publicados de 2009 até o presente momento.

Tabela 2. Escala de Sonolência de Epworth.

Numere de 0 a 3 quais são as chances de cochilar

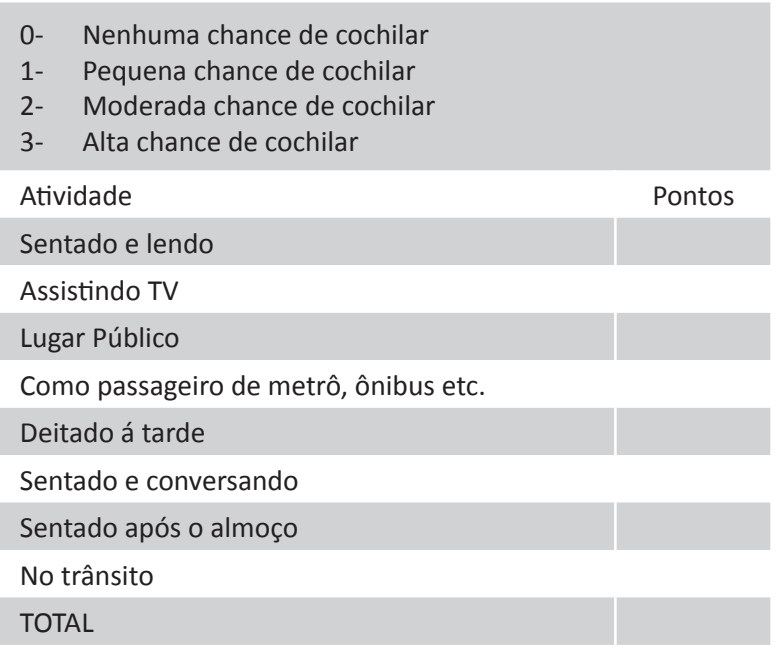

\section{Escala do Ronco de Stanford}

Usada para avaliar o ronco, nesta escala, o pesquisador dá uma nota de 0 a 10, de acordo com a intensidade do mesmo, sendo esta referida por parceiros. Ela permite a detecção do ronco e a sua classificação em cinco categorias de acordo com o valor da nota, que deve ser correspondente ao incômodo que este gera para o parceiro de cama, conforme descrito na figura 1. A grande desvantagem dessa escala é que pessoas sem parceiros de cama ou que não durmam junto, não podem ser avaliadas. ${ }^{10}$ Foi utilizada em um trabalho realizado pelo Hospital de Clínicas da Universidade de São Paulo (USP) de Ribeirão Preto para avaliar a efetividade do tratamento da SAOS através da uvuloplastia a laser, apresentando grande utilidade para comparação entre a intensidade do ronco do pré-operatório e após o ato cirúrgico. Neste trabalho, após o tratamento cirúgico, houve melhora do ronco em $76 \%$ dos pacientes, com redução da média da escala de Stanford de 8 para $2 .{ }^{11}$

O Questionário de Berlim é uma das ferramentas mais conhecidas e utilizadas no rastreio da SAOS. Tal questionário foi elaborado na conferência sobre patologia respiratória do sono em cuidados de saúde primários, na cidade de Berlim, na Alemanha, em abril de 1996 e validado por Netzer e colaboradores em 1999.12 $\mathrm{Na}$ ocasião, apresentou bons valores de sensibilidade e especificidade para identificação da SAOS (86\% e 77 \%, respectivamente, para um Índice de Apneia/Hipopneia (IAH) - encontrado através PSG - superior a 5, 54\% e 97 $\%$ para um IAH superior a 15 e $17 \%$ e $97 \%$ para um IAH maior do que 30$)^{13}$

A identificação do alto risco de SAOS se baseia em uma série de perguntas autoaplicáveis, divididas em três categorias, ilustradas na figura 2. A Categoria 1 se refere à presença de ronco, na qual o paciente avalia a intensidade do ronco e sua frequência. Na Categoria 2,o questionamento é a respeito de episódios de sonolência diurna e adormecimento durante atividades cotidianas. Por fim, na Categoria 3, pergunta-se sobre história de Hi-

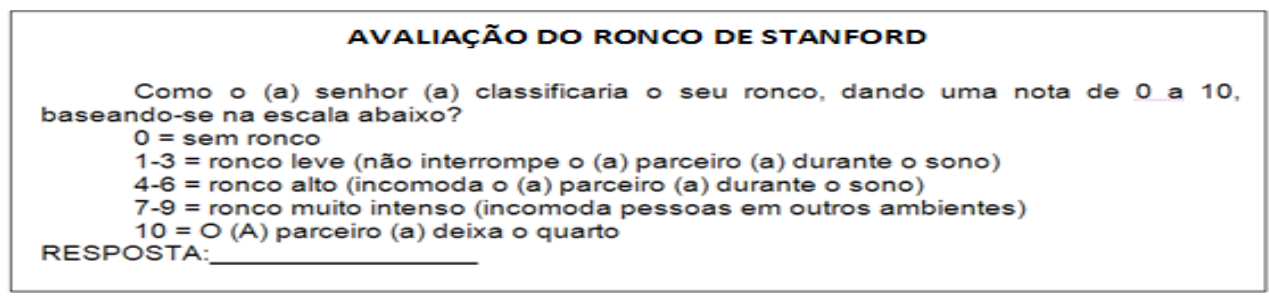

Figura 1. Escala do Ronco de Stanford. 
pertensão Arterial Sistêmica (HAS) e calcula-se o Índice de Massa Corporal (IMC). A interpretação do questionário idealizada por Netzer preconiza que as categorias devem ser pontuadas separadamente, somando 1 ponto a cada resposta positiva em que os sintomas sejam frequentes. As categorias 1 e 2 serão consideradas positivas quando obtiverem pontuação total maior ou igual a 2 . A categoria 3 é considerada positiva se o paciente tiver
HAS ou obesidade (IMC superior ou igual a $30 \mathrm{~kg} / \mathrm{m}^{2}$ ). São classificados como "alto risco" para SAOS todos aqueles que tiverem duas ou mais categorias positivas. ${ }^{13}$

Desde então, diversos estudos epidemiológicos vêm utilizando o questionário como método de triagem na população geral, traduzindo-o e validando-o em diversos idiomas. Temos a versão em português validada por Vaz e seus colaboradores, em 2011..$^{14}$ Além disso,

\begin{tabular}{|c|c|}
\hline \multicolumn{2}{|c|}{ Questionário Clínico de Berlim } \\
\hline Categoria 1 & Categoria 2 \\
\hline 4. Você ronca? & \multirow{2}{*}{$\begin{array}{l}\text { 1. Quantas vezes você se sente cansado ou com fadiga } \\
\text { depois de acordar? }\end{array}$} \\
\hline ( ) Sim & \\
\hline ( ) Não & Praticamente todos os dias \\
\hline ( ) Não sei & 3-4 vezes por semana \\
\hline & 1-2 vezes por semana \\
\hline 5. Seu ronco é: & Nunca ou praticamente nunca \\
\hline Pouco mais alto que sua respiração? & \multirow{2}{*}{$\begin{array}{l}\text { 2. Quando vc está acordado você se sente cansado, } \\
\text { fadigado ou não sente bem? }\end{array}$} \\
\hline Tão mais alto que sua respiração? & \\
\hline Mais alto do que falando? & \multirow{2}{*}{$\begin{array}{l}\text { Praticamente todos os dias } \\
3-4 \text { vezes por semana }\end{array}$} \\
\hline $\begin{array}{l}\text { Muito alto que pode ser ouvido nos quartos próxi- } \\
\text { mos? }\end{array}$ & \\
\hline & \multirow{4}{*}{$\begin{array}{l}\text { 1-2 vezes por semana } \\
\text { Nunca ou praticamente nunca } \\
\text { 3. Alguma vez você cochilou ou caiu no sono enquanto } \\
\text { dirigia? }\end{array}$} \\
\hline 6. Com que freqüência você ronca? & \\
\hline Praticamente todos os dias & \\
\hline 3-4 vezes por semana & \\
\hline $1-2$ vezes por semana & ( ) $\operatorname{Sim}$ \\
\hline Nunca ou praticamente nunca & ( ) Não \\
\hline 7. O seu ronco incomoda alguém? & \multirow{2}{*}{ Categoria 3} \\
\hline ( ) Sim & \\
\hline ( ) Não & \multirow{5}{*}{$\begin{array}{l}\text { 9. Você tem pressão alta? } \\
\text { ( ) Sim } \\
\text { ( ) Não } \\
\text { ( ) Não sei } \\
\text { IMC= }\end{array}$} \\
\hline 8. Alguém notou que você para de respirar en- & \\
\hline quanto dorme? & \\
\hline Praticamente todos os dias & \\
\hline 3-4 vezes por semana & \\
\hline \multicolumn{2}{|l|}{$1-2$ vezes por semana } \\
\hline \multicolumn{2}{|l|}{ Nunca ou praticamente nunca } \\
\hline \multicolumn{2}{|l|}{ Pontuação das perguntas: } \\
\hline \multicolumn{2}{|l|}{ Qualquer resposta circulada é considerada positiva } \\
\hline \multicolumn{2}{|c|}{ Pontuação das categorias: } \\
\hline \multicolumn{2}{|c|}{ Categoria 1 é positiva com 2 ou mais respostas positivas para as questões 1-5 } \\
\hline \multicolumn{2}{|c|}{ Categoria 2 é positiva com 2 ou mais respostas positivas para as questões $6-8$} \\
\hline \multicolumn{2}{|c|}{ Categoria 3 é positiva se a resposta para a questão 9 é positiva ou o IMC $>30$} \\
\hline \multicolumn{2}{|c|}{ Resultado final: } \\
\hline \multicolumn{2}{|l|}{2 ou mais categorias positivas indica alto risco para AOS } \\
\hline
\end{tabular}

Referência: Netzer NC, Stoohs RA, Netzer CM, Clark K, Strohl KP. Using the Berlin Questionnaire to identify patients at risk for the sleep apnea syndrome. Ann Intern Med. 1999 Oct 5;131(7):485-91.

Figura 2. Questionário de Berlim. 


\section{Artigo de revisão}

tem se buscado abranger a aplicação das perguntas em pacientes de cuidados secundários. No entanto, nestes casos, o questionário não tem se mostrado tão eficiente, com especificidade e sensibilidade mais baixas. $\mathrm{Na}$ pesquisa supracitada, o questionário mostrou-se com graus diferentes de sensibilidade e especificidade para cada doença. Por exemplo, o grupo de pacientes com fibrilação arterial a sensibilidade foi de $86 \%$ e a especificidade de $89 \%$, enquanto que para os pacientes com HAS resistente estes valores ficaram em 85,5\% e 65\%, respectivamente. Vale ressaltar, ainda, que, ao classificar a gravidade na doença, os pesquisadores encontraram decréscimo progressivo dos valores de sensibilidade, conforme se aumentava o IAH. ${ }^{14}$

Estudos mais recentes, como o de Ulasli e colaboradores, publicado em Julho de 2014, realizado com 1.450 pacientes de uma clínica especializada em sono, mostraram o questionário de Berlim com sensibilidade de 73\% e especificidade de 44\%, concluindo, desta forma, que este não é um bom preditor de AOS. ${ }^{15}$

Apesar das falhas apontadas pela literatura, o Questionário de Berlim ainda é um instrumento de grande valia para a detecção de SAOS, tendo em vista as limitações financeiras e espaciais do exame padrãoouro. No Brasil, são poucos os lugares onde este exame é realizado pelo Sistema Único de Saúde. Sendo assim, esta ferramenta é um bom recurso para priorizar os pacientes com mais fatores de risco associados.

Tabela 3. Mnemônico "STOP-BANG", com sua tradução para o português. STOP-BANG (traduzido).

\begin{tabular}{|l|l|}
\hline \multicolumn{1}{|c|}{ Tópico (em inglês): } & \multicolumn{1}{|c}{ Pergunta correspondente: } \\
\hline 1) Snoring & Você ronca alto? \\
\hline 2) Tiredness & $\begin{array}{l}\text { Você se sente frequentemente } \\
\text { cansado ao longo do dia? }\end{array}$ \\
\hline 3) Observed apnoea & $\begin{array}{l}\text { Alguém já lhe disse que você parou } \\
\text { de respirar durante o sono? }\end{array}$ \\
\hline 4) (blood) Pressure & $\begin{array}{l}\text { Você trata ou já realizou tratamen- } \\
\text { to para Hipertensão Arterial? }\end{array}$ \\
\hline 5) Body Mass Index & $\begin{array}{l}\text { Possui um Índice de Massa Corpo- } \\
\text { ral superior a } 35 \text { kg/m²? }\end{array}$ \\
\hline 6) Age & Possui idade superior a 50 anos? \\
\hline $\begin{array}{l}\text { 7) Neck Circunfer- } \\
\text { ence }\end{array}$ & $\begin{array}{l}\text { Possui circunferência cervical supe- } \\
\text { rior a } 40 \text { centímetros? }\end{array}$ \\
\hline 8) Gender & É do sexo masculino? \\
\hline
\end{tabular}

\section{STOP-BANG Questionnaire}

Este questionário foi elaborado na Universidade de Toronto, no Canadá, tendo sido utilizado amplamente em diversos países desde 2008. ${ }^{16}$ É um método de fácil aplicação, autoaplicável, que consiste em uma série de oito perguntas, cujas respostas são apenas sim ou não. ${ }^{17}$ Foi desenvolvido a partir de variáveis já reconhecidas como associadas a SAOS. Incialmente, era aplicada apenas em pacientes em pré-operatório pelos anestesistas. $\mathrm{O}$ nome deste questionário é justamente um mnemônico que se refere à letra inicial de cada tópico abordado através das oito indagações. ${ }^{18}$ Os tópicos abordados e suas perguntas correspondentes estão esquematizados na tabela 3. A presença de 3 ou mais respostas afirmativas indica um alto risco para SAOS, enquanto apenas 2 ou menos respostas positivas indicam um baixo risco. ${ }^{19}$

Diversos estudos têm comparado a sensibilidade e a especificidade deste questionário para identificação de SAOS. Pesquisas canadenses, norte-americanas e asiáticas demonstraram sensibilidade de 82,6-86,1\%, 87-92,9\% e 95,6-100\%, respectivamente, em pacientes com SAOS leve, moderada e acentuada, sendo tal gravidade determinada pelo valor do IAH. No entanto, a especificidade deste questionário é pequena, sendo de $40,4-47 \%$ e $35-37 \%$ nos casos de SAOS moderada e acentuada, respectivamente, demonstrando um baixo valor preditivo positivo (42-60,8\% e 31-43,5\% para SAOS moderada e grave). ${ }^{18-22} \mathrm{O}$ valor preditivo negativo, por sua vez, foi mensurado em 81,8-90,2\% e 93,4-100\% nos pacientes com SAOS moderada e acentuada, respectivamente. ${ }^{18-22}$ Foi realizada recentemente uma pesquisa na cidade de São Paulo, com aplicação deste questionário na população geral. A sensibilidade e o valor preditivo negativo encontrados estiveram dentro da faixa percentual descrita acima. ${ }^{19}$

Em pesquisa norte-americana recente, quando comparado com o Questionário de Berlim, o STOPBANG apresentou sensibilidade (68\% contra 57\%) e especificidade ( $49 \%$ contra $41 \%$ ) maiores em pacientes com SAOS leve. ${ }^{19}$ Em outro estudo mais amplo, realizado em laboratório canadense de medicina do sono, o STOP-BANG apresentou sensibilidade maior do que o Questionário de Berlim nos casos leves (90\% vs. 86\%), nos casos moderados (93\% vs. $91 \%$ ) e na SAOS grave ( $96 \%$ vs. $89 \%$ ). Também apresentou especificidade superior $(42 \%, 28 \%$ e $21 \%$, respectivamente, contra $25 \%$, $28 \%$ e $18 \%)$, maior valor preditivo negativo $(29,4 \%$, $64,7 \%$ e $88,2 \%$, respectivamente, contra $15,8 \%, 57,9 \%$ e $67,4 \%$ ) e valores preditivos positivos maiores $(93,7 \%$, 
$73,9 \%$ e $48,6 \%$, respectivamente, contra $91,7 \%, 73,4 \%$ e $45,9 \%$ ), nos três grupos de gravidade da SAOS. ${ }^{23}$

Quando aplicado em diferentes populações, a presença de cinco ou mais respostas positivas no contexto pré-operatório indicou uma alta probabilidade deSAOS moderada ou acentuada, apontando que a aplicação deste como rotina pré-operatória é útil tanto para o diagnóstico e tratamento da SAOS (especialmente de SAOS oculta), quanto para o ajuste de medicação anestésicas indutoras do sono. ${ }^{18}$ Além disso, em uma pesquisa de pré-operatório de cirurgias eletivas otorrinolaringológicas, a quantidade de respostas positivas ao STOP-BANG foi diretamente proporcional ao percentual de complicações pré-operatórias, que muitas vezes poderiam ser evitadas ou atenuadas caso a presença de DRS fosse detectada anteriormente ao ato cirúrgico. ${ }^{17}$ Em populações atendidas em centros norte-americanos de medicina do sono, por outro lado, tem se mostrado que este questionário é pouco útil, já que seu principal valor é baseado na capacidade de afastar quem de fato não possui SAOS (alto valor preditivo negativo) e a população que procura esses centros possui uma prevalência maior de SAOS do que outras populações estudadas. ${ }^{19,22}$ Por outro lado, em centros asiáticos de medicina do sono, tal questionário tem sido apontado como de grande utilidade pra triagem de SAOS, especialmente por sua grande sensibilidade e alto valor preditivo negativo, auxiliando assim na redução de custos com exames desnecessários. ${ }^{20} \mathrm{Em}$ pacientes com Doença Renal Crônica, oSTOP-BANG demonstrou alta sensibilidade e um grande valor preditivo negativo, porém apresentou uma especificidade bem abaixo da desejável, não sendo de grande utilidade para a investigação de DRS nesses pacientes. ${ }^{24}$

Assim, na população geral e pré-operatória (eletiva), a maioria das pesquisas atuais sugerem que pacientes com 3 ou mais respostas positivas no STOP-BANG se beneficiariam com a realização de PSG..$^{18-20}$ Entretanto, outros estudos afirmam que o principal fator para indicar a realização de PSG é a intensidade do quadro clínico, especialmente em centros de medicina dosono, não havendo diferença no número de indicações deste exame após a aplicação do STOP-BANG.19,22 Ainda não há consenso na literatura sobre quando deve ser indicada ou não a PSG através da resposta a este questionário em cada tipo de população.

\section{Sleep Apnea Quality Life Index (SAQLI)}

Este questionário foi criado em 1998, por Flemons \& Reimer, e adaptado para o português de Portugal em
2008, por Sampaio, Pereira e Winck. ${ }^{25}$ É um instrumento específico amplamente utilizado para a pesquisa de qualidade de vida na SAOS, dada a prevalência da doença e consequente perturbação das funções físicas, sociais e psicológicas. Utilizado quando a preocupação é com o sucesso do tratamento e/ou com os efeitos colaterais. Avalia 4 domínios da QV fundamentais ou específicos dos doentes com SAOS como demonstrados na tabela 4 .

Pode ter um domínio adicional quando se avalia o tratamento cirúrgico ou com CPAP. Seria o $5^{\circ}$ domínio com 5 Itens. Cada questão é pontuada com variações de 1 a 7 , onde 1 significa muito grande e 7 nenhum. Para cada um dos 4 domínios a média do resultado é obtida dividindo-se a soma de todos os itens, dentro do domínio, pelo número total de itens do domínio. A pontuação total é calculada pela soma dos 4 domínios dividida por 4. O resultado é interpretado sendo 1 menor QV e 7 maior QV. Uma desvantagem deste instrumento é o fato de não ser autoaplicável, necessitando a presença do pesquisador.

\section{Considerações finais}

Na experiência do grupo de pesquisa do LabSono HUGG, nos pacientes que realizaram PSG e responderam aos Questionários de Berlim eSTOP-BANG, encontramos maior sensibilidade e especificidade no STOPBANG quando comparado ao Berlim, especialmente nos casos mais graves, apresentando sensibilidade de $87,50 \%$ na SAOS leve e de $100 \%$ na SAOS moderada e grave, com aumento significativo da positividade do questionário conforme se elevou o valor do IAH $(\mathrm{p}<0,01)$. Uma dificuldade prática, no entanto, foi a aferição da circunferência cervical. Fator facilmente contornável em clínicas em geral pela fácil disponibilidade de fitas métricas, em nossas avaliações isto dificultou a autoaplicação do questionário, sugerida por seus idealizadores. Além disso, outro impasse é a baixa escolaridade da população estudada o que se tornou um complicador na compreesão da linguagem escrita.

Tabela 4. Quatros domínios do SAQLI e quantidade de itens.

\begin{tabular}{l|c}
\hline 1 - Funcionamento diário & $\mathbf{1 1}$ Itens \\
\hline 2 - Interação social & 13 Itens \\
\hline 3 - Funcionamento emocional & 11 Itens \\
\hline 4 - Sintomas relacionados a doença & 5 Itens \\
\hline
\end{tabular}




\section{Artigo de revisão}

Em relação ao Questionário de Berlim encontramos dificuldade para responder as perguntas 8 e 9, pois, especialmente as mulheres, não sabiam dirigir ou não possuíam automóvel.

Os questionários específicos para SAOS estão sendo usados cada vez mais nos estudos mais recentes e, gradualmente, substituindo as escalas genéricas. ${ }^{26}$ Portanto, embora ainda sejam necessárias mais pesquisas em diferentes populações para se avaliar a atuação do STOP-BANG e dos demais questionários na detecção e triagem da SAOS, pode-se afirmar que estas são ferramentas de grande utilidade diagnóstica e que, junto com a avaliação clínica feita por profissional especializado, é possível que a realização de PSG seja indicada de forma mais precisa, visto que tal exame apresenta alto custo e alta complexidade, sendo ainda pouco disponível, especialmente no Brasil.

\section{Referências}

1. Viegas CAA. Epidemiologia dos distúrbios respiratórios do sono. J Bras Pneumol. 2010;36(2):S1-S61.

2. Phillipson EA. Sleep apnea - A major public health problem (editorial). N Engl J Med. 1993;328(17):1271-3. http://dx.doi. org/10.1056/NEJM199304293281712

3. Drager LF, Ladeira RT, Brandão-Neto RA, et al. Síndrome da Apnéia Obstrutiva do Sono e sua Relação com a Hipertensão Arterial Sistêmica - Evidências Atuais. Arq Bras Cardiol. 2002;78:531-6.

4. Shahid A, Wilkinson K, Marcu S, et al. Stop, That and one hundred other sleep scales. Springer: New York; 2012. http:// dx.doi.org/10.1007/978-1-4419-9893-4

5. Cook Y, Schmitt F, Berry D, et al. The effects of nocturnal sleep, sleep disordered breathing and periodic movements of sleep on the objective and subjective assessment of daytime somnolence in healthy aged adults. J Sleep Res. 1988;17:95.

6. Johns MW. A New Method for Measuring Daytime Sleepiness: The Epworth Sleepiness Scale. Sleep Med. 1991;14:540-5.

7. Boari L, Cavalcanti CM, Bannwart SRFD, et al. Avaliação da escala de Epworth em pacientes com a Síndrome da apnéia e hipopnéia obstrutiva do sono. Rev Bras Otorrinolaringol 2004; 70 (6): 752-756.

8. Johns MW. Daytime Sleepiness, Snoring and Obstructive Sleep Apnea: The Epworth Sleepiness Scale. Chest. 1993;103(1):306. http://dx.doi.org/10.1378/chest.103.1.30

9. Bertolazi AN, Fagondes SC, Hoff LS, et al. Validação da Escala de Sonolência de Epworth em português para uso no Brasil. J Bras Pneumol. 2009;35(9):877-883.

10. Rodrigues MM, Dibbern RS, Goulart CWK. Obstrução nasal e alto escore de Mallampati como fatores de risco associados para Apneia Obstrutiva do Sono. Rev Bras Otorrinolaringol. 2010;76(5):596-9.

11. Thuler ER, Dilbert RS, Fomin DS, et al. Uvulopalatoplastia a laser - Análise comparativa da melhora clínica e dos critérios de indicação. Rev Bras Otorrinolaringol. 2002;68(2):190-3.

12. Hiestand DM, Britz P, Goldman M, et al. Prevalence of Symp- toms and Risk of Sleep Apnea in the US Population: Results From the National Sleep Foundation Sleep in America 2005 Poll. Chest. 2006;130(3):780-786. http://dx.doi.org/10.1378/ chest.130.3.780

13. Netzer NC, Stoohs RA, Netzer CM, et al. Using the Berlin Questionnaire to identify patients at risk for the sleep apnea syndrome. Ann Intern Med. 1999; 131(7):485-491. http://dx.doi. org/10.7326/0003-4819-131-7-199910050-00002

14. Vaz AP, Drummond M, Mota PC, et al. Tradução do Questionário de Berlim para língua Portuguesa e sua aplicação na identificação da SAOS numa consulta de patologia respiratória do sono. Rev Port Pneumol. 2011;17(2):59-65.

15. Ulasli SS, Gunay E, Koyuncu T, et al. Predictive value of Berlin Questionnaire and Epworth Sleepiness Scale for obstructive sleep apnea in a sleep clinic population. Clin Respir J. 2014;8(3):292-6. http://dx.doi.org/10.1111/crj.12070

16. Reis R, Teixeira F, Martins V, et al. Validation of a Portuguese Version of the STOP-Bang Questionnaire as a Screening Tool for Obstructive Sleep Apnea: Analysis in a Sleep Clinic. Rev Port Pneumol. 2015;21:61-8.

17. Vasu TS, Doghramji K, Cavallazzi R, et al. Obstructive Sleep Apnea Syndrome and Postoperative Complications Clinical Use of the STOP-BANG Questionnaire. Arch Otolaryngol Head Neck Surg. 2010;136(10):1020-4.

18. Chung F, Subramanyam R, Liao P, et al. High STOP-Bang score indicates a high probability of obstructive sleep apnoea. Br J Anaesth. 2012;108(5):768-775.

19. Boynton $G$, Vahabzadeh A, Hammoud S, et al. Validation of the STOP-BANG Questionnaire among Patients Referred for Suspected Obstructive Sleep Apnea. J Sleep Disor: Treat Care. 2013;2:4.

20. Ong TH, Raudha S, Fook-Conhg S, et al. Simplifying STOPBANG: use of a simple questionnaire to screen for OSA in an Asian population. Sleep Breath. 2010;14(4):371-6. http://dx.doi. org/10.1007/s11325-010-0369-9

21. Silva GE, Vana KD, Goodwin JL, et al. Identification of Patients with Sleep disordered Breathing: Comparing the four-Variable Screening Tool, STOP, STOP-Bang, and Epworth Sleepiness Scales. J Clin Sleep Med. 2011;7(5):467-472.

22. Farney RJ, Walker BS, Farney RM, et al. The STOP-Bang Equivalent Model and Prediction of Severity of Obstructive Sleep Apnea: Relation to Polysomnographic Measurements of the Apnea/Hypopnea Index. J Clin Sleep Med. 2011;7(5):459465. http://dx.doi.org/10.5664/jcsm.1306

23. Pereira EJ, Driver HS, Fitzpatrick MF. Comparing a Combination of Validated Questionnaires and Level III Portable Monitor with Polysomnography to Diagnose and Exclude Sleep Apnea. J Clin Sleep Med. 2013;9(12):1259-1266. http://dx.doi. org/10.5664/jcsm.3264

24. Nicholl DDM, Ahmed SB, Hanly PJ, et al. Diagnostic Value of Screening Instruments for Identifying Obstructive Sleep Apnea in Kidney Failure. J Clin Sleep Med. 2013;9(1):31-8.

25. Sampaio RS, Pereira MG, Winck JC. Adaptacção Portuguesa do Questionário de Qualidade de Vida (SAQLI) nos doentes com Síndrome de Apneia Obstrutiva do Sono. Rev Port Pneumol. 2012;18(4):166-174.

26. Stucki A, Cieza A, Schuurmans MM, et al. Content comparison of health-related quality of life instruments for obstructive sleep apnea. Sleep Med. 2008;9:199-206. 